Check for updates

Cite this: RSC Adv., 2017, 7, 27105

Received 29th March 2017

Accepted 8th May 2017

DOI: 10.1039/c7ra03635c

rsc.li/rsc-advances

\section{Controlled preparation of Ag nanoparticles on graphene with different amount of defects for surface-enhanced Raman scattering}

\author{
Jun Qin, Lujun Pan, (D) Chengwei Li, Lichen Xia, Nan Zhou, Yingying Huang \\ and Yi Zhang
}

Graphene with different amounts of defects was prepared by chemical vapor deposition by controlling the flow rate of hydrogen, on which Ag nanoparticles (NPs) were deposited by magnetron sputtering. It is certified that the defects mainly consist of $\mathrm{C}-\mathrm{O}$ bonds that affect the morphology and distribution of the prepared Ag NPs. The Ag NPs/graphene composition exhibits good surface-enhanced Raman scattering activity, which is largely increased by increasing the percentage of the functional group $\mathrm{C}-\mathrm{O}$ in graphene.

\section{Introduction}

Surface-enhanced Raman scattering (SERS) has been used as a powerful tool for the detection of the structures of molecules in many fields such as materials, biosensing, chemistry, and physics. ${ }^{\mathbf{1 - 6}}$ It has attracted significant attention since SERS was first discovered on a rough Ag surface by Fleischmann in $1974 .^{7}$ As is well-known, SERS results from a combination of electromagnetic enhancement mechanisms (EM) and chemical enhancement mechanisms (CM) ${ }^{8-10}$ The EM originates from the surface plasmon resonance, which is originated from metal NPs, and the CM is caused by charge transfer between the probe materials and the substrates. ${ }^{11,12}$ To date, noble metals, such as $\mathrm{Au}, \mathrm{Ag}$, and Pt, have been widely utilized for SERS. ${ }^{13}$ Han et al. found that $\mathrm{Ag}$ showed a better SERS activity as compared to other metals. ${ }^{14}$ Malynych et al. indicated that Ag NPs more efficiently interacted with light than any other particles with the same dimensions. ${ }^{15}$ Moreover, Kha et al. have reported that Ag is the only material whose plasmon resonance can be tuned to any wavelength in the visible spectrum. ${ }^{16}$ However, Ag NPs are easily oxidized in air, and they have a high fluorescent background and low adsorption of the probe molecules, which will limit their SERS activity. ${ }^{\mathbf{1 7}}$ Therefore, finding an ideal substrate for SERS becomes most desirable.

Graphene, a two-dimensional lattice of carbon with only one atom layer, has been a hot research topic because of its excellent optical, electronic, and mechanical properties. ${ }^{18-20}$ Especially, graphene enables charge transfer with probe materials through the $\pi-\pi$ bonds. Recently, metals and graphene hybrids were used as SERS substrates, where graphene could absorb probe molecules, protect metals from oxidation, and quench

School of Physics and Optoelectronic Technology, Dalian University of Technology, No. 2 Linggong Road, Ganjingzi District, Dalian 116024, PR China. E-mail: lpan@dlut. edu.cn fluorescence from the metals. ${ }^{21-23}$ For example, Zhang et al. covered metal NPs with graphene, which served as a barrier to preserve the metal NPs and effectively suppressed the photobleaching effect. ${ }^{24} \mathrm{Li}$ et al. designed a system by introducing graphene as a subnanospacer between $\mathrm{Ag}$ NPs and the $\mathrm{Ag}$ film, which showed a high SERS intensity since graphene strengthened the ability to efficiently interact with probe molecules and quench the fluorescence background. ${ }^{25}$

Developing a graphene-Ag NPs hybrid that combines the properties of CM enhancement and EM enhancement is applicable for SERS. Recently, it has been widely studied that how the structures of this hybrid affect the SERS activity. For instance, Leem et al. introduced a three-dimensional graphenemetal hybrid that exhibited higher detection sensitivity of the probe material as compared to the uniform graphene-metal substrates. ${ }^{26} \mathrm{Fu}$ et al. developed a system with Ag NPs by sputtering and photochemical catalysis to prove that different densities of Ag NPs can create different Raman intensities. ${ }^{27}$ Wang et al. enlarged the surface area and modified the morphologies of the metals to obtain stronger SERS intensity. ${ }^{28}$ In these researches, significant attention was paid to change the structures of the metal materials and graphene hybrids. In fact, graphene itself when used as an SERS platform possesses different defects and layers, ${ }^{29,30}$ which can affect the SERS activity. However, few researchers have reported that the defects in graphene affect the SERS activity of the hybrids.

In this study, graphene with different percentages for $\mathrm{C}-\mathrm{O}$ was grown on a $\mathrm{Cu}$ foil by chemical vapor deposition (CVD), and different amounts of $\mathrm{C}-\mathrm{O}$ were produced on it by controlling the flow rate of hydrogen during the reaction. Ag NPs were sputtered on graphene for SERS; the different amounts of $\mathrm{C}-\mathrm{O}$ on graphene can lead to the divergence of $\mathrm{Ag}$ distribution. The relationship between the $\mathrm{C}-\mathrm{O}$ in graphene and SERS activity was investigated. Statistical results indicated that the prepared 
graphene and $\mathrm{Ag}$ NPs obviously exhibited diverse Raman enhancement for rhodamine 6G molecules.

\section{Experimental}

\subsection{Preparation of graphene}

Graphene sheets were grown on copper foils (25 $\mu \mathrm{m}$ thickness and $99.8 \%$ purity, Alfa Aesar) by CVD. ${ }^{31}$ In a typical process, $\mathrm{Cu}$ foils were cleaned by ultrasonication in an acetone solution, ethanol, and deionized water for $15 \mathrm{~min}$, successively. After cleaning, $\mathrm{Cu}$ foils were placed into a quartz tube before the quartz tube was vacuumed $\left(10^{-1} \mathrm{~Pa}\right)$ to remove oxygen. Then, the tube was filled with a mixed gas of $\mathrm{H}_{2}$ and Ar with the flow rates of 100 and $700 \mathrm{sccm}$, respectively. After this, the temperature of the quartz tube was increased from room temperature to $1050{ }^{\circ} \mathrm{C}$. Before the reaction, the temperature in the tube was decreased to $1000{ }^{\circ} \mathrm{C}$ and the flow rates of $\mathrm{H}_{2}$ and $\mathrm{Ar}$ were changed to $X$ ( $X$ ranges from 10 to $500 \mathrm{sccm}$ ) and $1000 \mathrm{sccm}$, respectively. Then, methane $\left(\mathrm{CH}_{4}\right)$ with a flow rate of $9 \mathrm{sccm}$ was introduced to the quartz tube as a carbon source for $15 \mathrm{~min}$. Graphene with different amounts of defects was synthesized by adjusting the flow rate of $X$ for $\mathrm{H}_{2}$. At last, the samples were cooled down to room temperature under the protection of $\mathrm{Ar}$ and $\mathrm{H}_{2}$ with the flow rates of 1000 and $10 \mathrm{sccm}$, respectively. The samples were labeled as shown in Table 1.

\subsection{Deposition of the Ag NPs}

Graphene was transferred from $\mathrm{Cu}$ foils to Si substrate by the traditional method where samples were soaked in a $\mathrm{FeCl}_{3}$ solution to etch the $\mathrm{Cu}$ foils and washed with deionized water. ${ }^{32}$ $\mathrm{Ag}$ NPs were sputtered on the prepared graphene with different amount of defects by a vacuum magnetron sputtering system (JCP-200, BTSC563). The working pressure was 1 Pa under an Ar environment, and the sputtering power was $20 \mathrm{~W}$. The sizes of the Ag NPs were optimized by altering the sputtering time from 15 to $20 \mathrm{~s}$.

\subsection{SERS measurements}

R6G with different concentrations (from $10^{-5}$ to $10^{-7} \mathrm{M}$ ) was selected as a probe molecule. In this experiment, $80 \mu \mathrm{l}$ of R6G

Table 1 Sample name and the corresponding gas mixtures in the graphene growth stage

Sample name

\section{$\mathrm{G}_{10}$}

$\mathrm{G}_{50}$

$\mathrm{G}_{75}$

$\mathrm{G}_{100}$

$\mathrm{G}_{150}$

$\mathrm{G}_{200}$

$\mathrm{G}_{250}$

$\mathrm{G}_{300}$

$\mathrm{G}_{400}$

$\mathrm{G}_{500}$
Flow rates of gases $\mathrm{Ar}: \mathrm{H}_{2}: \mathrm{CH}_{4}(\mathrm{sccm})$

$1000: 10: 9$ $1000: 50: 9$ $1000: 75: 9$ $1000: 100: 9$ $1000: 150: 9$ $1000: 200: 9$ $1000: 250: 9$ $1000: 300: 9$ $1000: 400: 9$ $1000: 500: 9$ was dripped on each substrate $\left(1 \times 1 \mathrm{~cm}^{2}\right)$. Raman spectra were obtained under the same conditions as follows: the exciting light source was a $532 \mathrm{~nm} \mathrm{He}-\mathrm{Ne}$ laser with an excitation power of $0.75 \mathrm{~mW}$, and the laser beam was focused by a $50 \times$ objective lens.

\subsection{Characterization}

The sizes and distributions of the Ag NPs on graphene were observed by a scanning electron microscope (SEM; FEI, NOVA NanoSEM 450). The prepared graphene was characterized by Xray photoelectron spectroscopy (XPS, ESCALAB 250xi) and Raman spectroscopy (Renishaw in Via plus, $532 \mathrm{~nm}$ ).

\section{Results and discussion}

\subsection{The characteristic of graphene with different flow rates of hydrogen}

Raman spectra for graphene prepared at different flow rates of hydrogen ranging from 10 to $500 \mathrm{sccm}$ are shown as Fig. 1a. The characteristic Raman bands of graphene at $\sim 1345 \mathrm{~cm}^{-1}$ (D band), $\sim 1585 \mathrm{~cm}^{-1}$ (G band), and $\sim 2685 \mathrm{~cm}^{-1}$ (2D band) can be clearly observed. However, each sample shows a different intensity of $\mathrm{D}$ band, $\mathrm{G}$ band, and $2 \mathrm{D}$ band, which are affected by the layers and defects of graphene. Amongst these spectra, the spectrum of sample $\mathrm{G}_{10}$ exhibits a higher $2 \mathrm{D}$ band and a smaller $D$ band, and it was concluded that the sample $G_{10}$ was thinnest as compared to other samples. Moreover, the intensity of the $2 \mathrm{D}$ band for the grown graphene decreased with the increasing flow rate of hydrogen. As is known, the $\mathrm{D}$ to $\mathrm{G}$ band intensity ratio $\left(I_{\mathrm{D}} / I_{\mathrm{G}}\right)$ is related to the quantity of defects on graphene. ${ }^{33}$ Fig. $1 \mathrm{~b}$ shows that the $I_{\mathrm{D}} / I_{\mathrm{G}}$ ratio for these graphene samples increases with the increasing flow rate of hydrogen. The ratio of $I_{\mathrm{D}} / I_{\mathrm{G}}$ reached its largest value when the flow rate of hydrogen was increased to $100 \mathrm{sccm}$, indicating that the sample $\mathrm{G}_{100}$ had more defects as compared to other samples. Fig. 1c shows the ratio of the $\mathrm{G}$ band to $2 \mathrm{D}$ band intensity $\left(I_{\mathrm{G}} / I_{2 \mathrm{D}}\right)$, which is related to the numbers of graphene layers. ${ }^{34}$ The $I_{\mathrm{G}} / I_{2 \mathrm{D}}$ ratio increased with the increasing flow rate of hydrogen, illustrating an increase in graphene layers. The $I_{\mathrm{G}} / I_{2 \mathrm{D}}$ for sample $\mathrm{G}_{10}, \mathrm{G}_{50}, \mathrm{G}_{75}$, $\mathrm{G}_{100}$, and $\mathrm{G}_{200}$ are $0.561,0.559,0.638,0.715$, and 0.915, respectively. It was estimated that the thicknesses for samples $\mathrm{G}_{10}, \mathrm{G}_{50}, \mathrm{G}_{75}$, and $\mathrm{G}_{100}$ are no more than 10 layers and the thickness for sample $\mathrm{G}_{200}$ is almost 20 layers. The $I_{\mathrm{G}} / I_{2 \mathrm{D}}$ ratios for the rest of the samples kept increasing with the increase of $\mathrm{H}_{2}$ flow rate. Moreover, the intensities of $\mathrm{G}$ band for the samples $\mathrm{G}_{200}, \mathrm{G}_{300}, \mathrm{G}_{400}$ and $\mathrm{G}_{500}$ are similar to those of the multilayer graphene. The layers of graphene continue to grow in the presence of more hydrogen. Therefore, graphene exhibits a few layers and contains abundant defects while using hydrogen less than $100 \mathrm{sccm}$ during the CVD growth. In this process, defects would be generated on graphene films, such as edges, grain boundaries, functional groups or structural disorders. ${ }^{35}$ The core density for growing graphene increased, and the grain sizes of graphene decreased ${ }^{36}$ with the increasing hydrogen flux from 10 to $100 \mathrm{sccm}$. Therefore, more grain boundaries and functional groups would be generated on the graphene film at a flow 

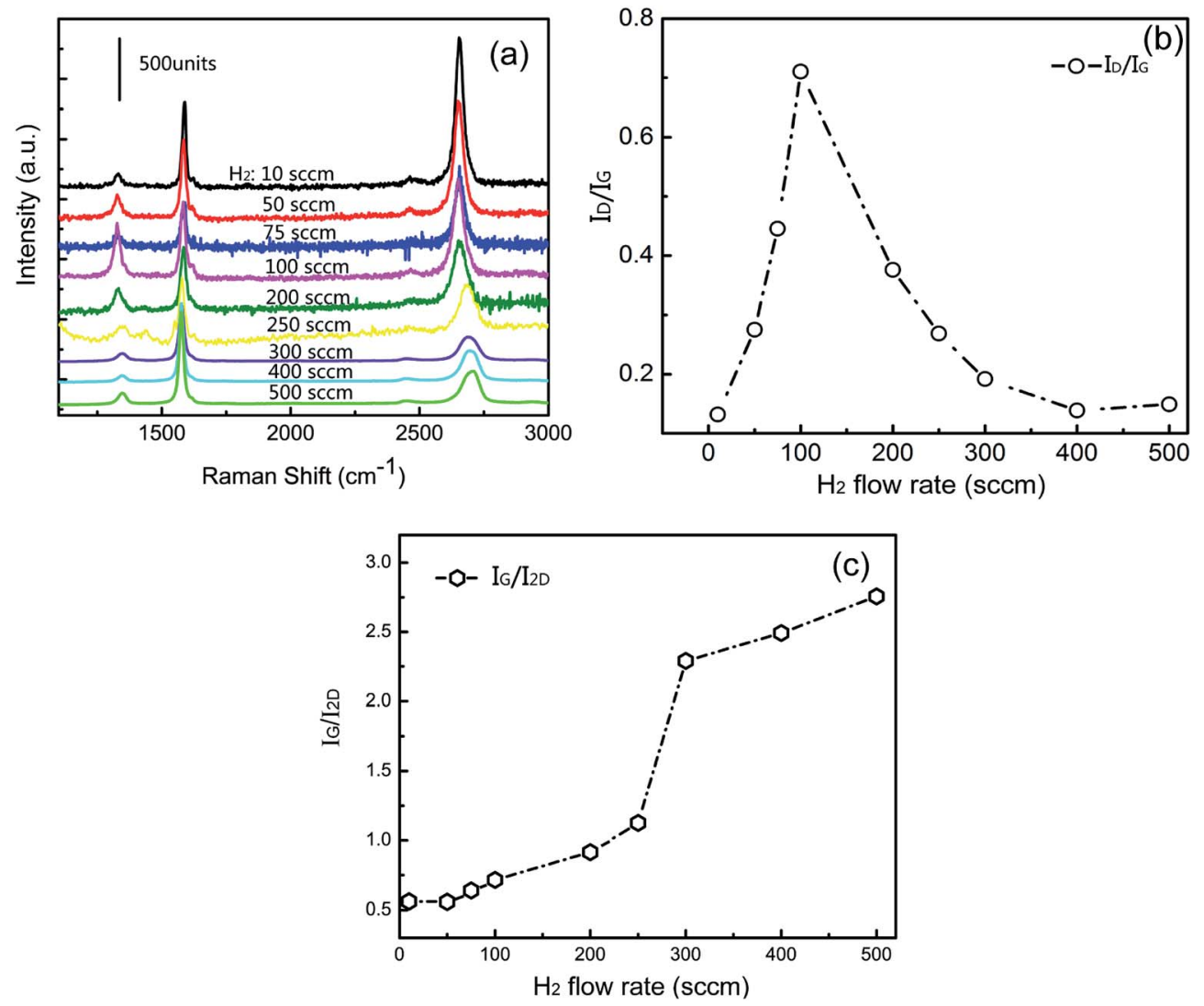

Fig. 1 (a) Raman spectra of graphene grown at different flow rates of $\mathrm{H}_{2}$. (b) $I_{\mathrm{D}} / I_{\mathrm{G}}$ ratio and $(\mathrm{c}) I_{\mathrm{G}} / I_{2 \mathrm{D}}$ of graphene grown at different flow rates of $\mathrm{H}_{2}$.

rate of $100 \mathrm{sccm}$. However, when the hydrogen flux exceeds 100 $\mathrm{sccm}$, the carbon source would be easily diffused and hydrogen would promote the fast removal of residual oxygen or contaminants from the surface. Furthermore, graphene grain sizes would saturate and the number of defects would decrease, such as oxygen-containing functional groups. ${ }^{37,38}$ Graphene enables charge transfer mainly owing to the functional groups, such as phenolic, carboxyl and epoxide groups, on the surface. ${ }^{39}$ Furthermore, these functional groups on graphene would provide active sites, which can absorb the $\mathrm{Ag}$ atoms or clusters to grow into bigger NPs. ${ }^{40}$ Considering this, XPS measurement was performed to determine the functional groups in these graphene films.

Fig. 2a-c show the C 1s XPS spectra for the samples $\mathrm{G}_{10}, \mathrm{G}_{100}$, and $\mathrm{G}_{200}$ on copper. These three C 1s XPS spectra are similar, and the $\mathrm{C} 1 \mathrm{~s}$ of graphene is fitted by four peaks, which include four kinds of carbon-containing functional groups: $\mathrm{C}=\mathrm{C}, \mathrm{C}-\mathrm{C}$, $\mathrm{C}-\mathrm{H}$, and $\mathrm{C}-\mathrm{O} . \mathrm{C}=\mathrm{C}$ bonds and $\mathrm{C}-\mathrm{C}$ bonds come from the structure of graphene, and $\mathrm{C}-\mathrm{H}$ bonds in graphene surface comes from the hydrogen etching process. In an argon protected environment, oxygen may have originated from oxides, such as $\mathrm{CuO}$ and $\mathrm{Cu}_{2} \mathrm{O}$, at the copper surface. During the reaction, these oxides are reduced into oxygen by hydrogen. Simultaneously, graphene is etched by the released oxygen, and then, the $\mathrm{C}-\mathrm{O}$ bonds are generated in graphene in this process. It was observed that the percentage of $\mathrm{C}-\mathrm{H}$ bonds was much less than that of $\mathrm{C}-\mathrm{O}$ bonds in each sample, indicating that $\mathrm{C}-\mathrm{H}$ would not obviously affect the distribution of the deposited $\mathrm{Ag}$ NPs. Fig. 2d shows that the percentage for $\mathrm{C}-\mathrm{O}$ bonds increased when the flow rate of hydrogen was increased from 10 to 100 sccm and then decreased when more hydrogen was introduced. Graphene grain sizes and grain boundaries would saturate when the flow rate of hydrogen is increased to $100 \mathrm{sccm}$. Functional groups, such as $\mathrm{C}-\mathrm{O}$ bonds, are mainly generated at grain boundaries. ${ }^{36}$ Thus, the amount of $\mathrm{C}-\mathrm{O}$ bonds would change at the air-graphene grain boundaries, which are limited by the flow rate of hydrogen. It is known that $\mathrm{C}-\mathrm{O}$ is electronegative and it tends to absorb the electropositive Ag atoms or clusters. This suggests that the electronegative $\mathrm{C}-\mathrm{O}$ in graphene would promote the formation of Ag NPs and affect the distribution and morphology of the Ag NPs. Thus, it is necessary to investigate the relationship between the percentage of $\mathrm{C}-\mathrm{O}$ and the formation of Ag NPs.

\subsection{The distribution and morphology for the Ag NPs on graphene}

As shown in Fig. 3a-g (the SEM images), Ag NPs were sputtered on graphene substrates with the same sputtering time. It was observed that the sizes and distances amongst Ag NPs are relatively uniform, but different in each sample. The sizes of Ag NPs become larger when the flow rate of hydrogen is increased 

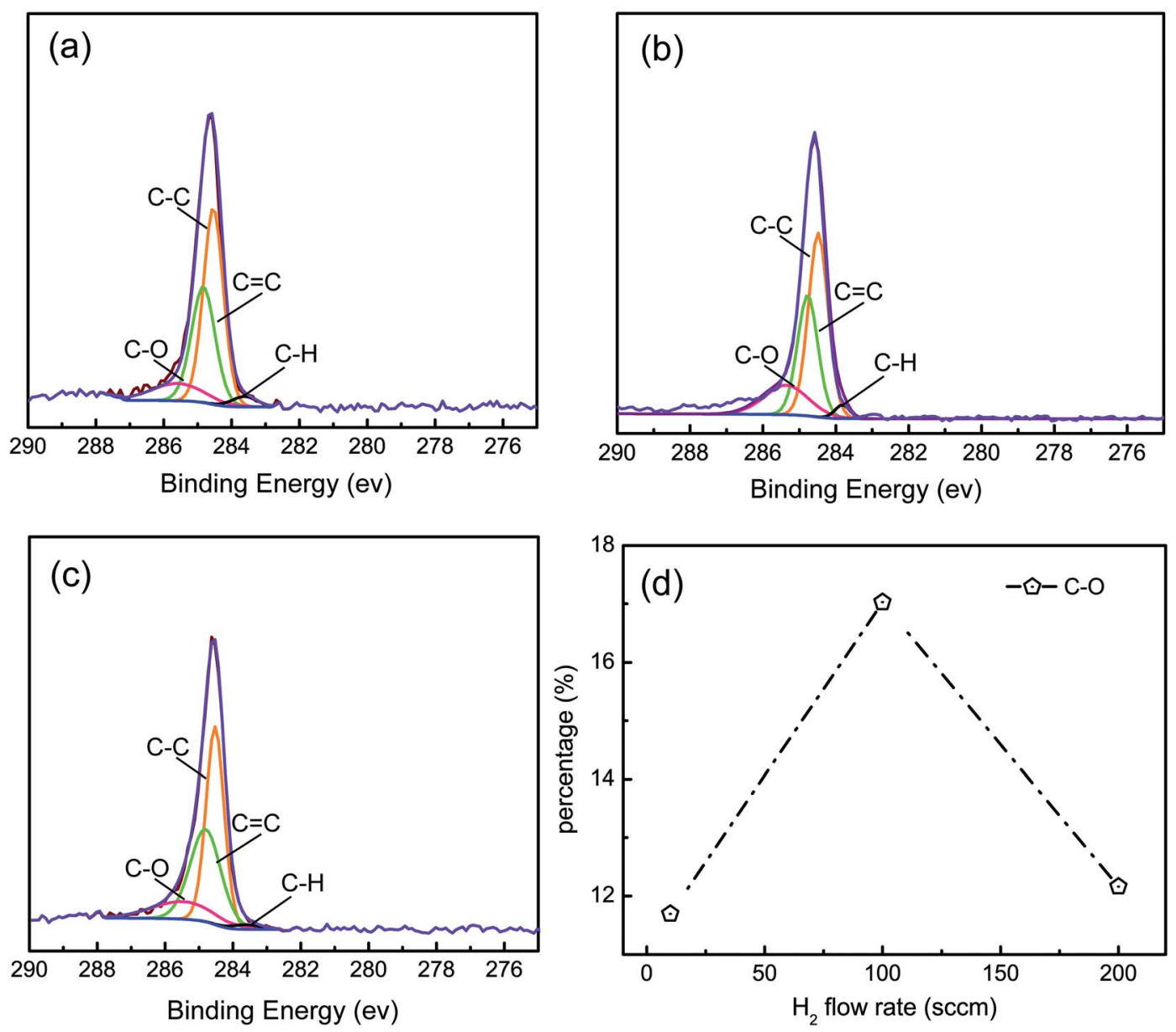

Fig. 2 C 1s XPS spectra for samples of (a) $G_{10}$, (b) $G_{100}$, and (c) $G_{200}$.

(d) The percentage of $\mathrm{C}-\mathrm{O}$ bonds for graphene.

from 10 to $100 \mathrm{sccm}$. The sizes of $\mathrm{Ag}$ NPs for the $\mathrm{G}_{100}-\mathrm{Ag}$ are larger than those of the rest of the samples, and these become more larger when the hydrogen flux exceeds $150 \mathrm{sccm}$. The heights of Ag NPs on $\mathrm{G}_{100}$ were detected by AFM, which ranged from 2 to $10 \mathrm{~nm}$. However, the distances between adjacent $\mathrm{Ag}$ NPs become smaller with the flow rate of hydrogen, which ranges from 10 to $100 \mathrm{~nm}$, and these become larger when the hydrogen flux exceeds $100 \mathrm{sccm}$. It was indicated that the average sizes for $\mathrm{Ag}$ NPs in the sample $\mathrm{G}_{100}$ were largest, but the distances were smallest. It is well known that the distance of less than $10 \mathrm{~nm}$ between adjacent particles is beneficial for forming hot spots for SERS. Fig. 3h shows the percentage of distances of less than $10 \mathrm{~nm}$ between $\mathrm{Ag}$ NPs calculated from the corresponding SEM images for the samples $\mathrm{G}_{10}-\mathrm{Ag}, \mathrm{G}_{50}-\mathrm{Ag}$, $\mathrm{G}_{100}-\mathrm{Ag}, \mathrm{G}_{150}-\mathrm{Ag}, \mathrm{G}_{200}-\mathrm{Ag}, \mathrm{G}_{250}-\mathrm{Ag}$, and $\mathrm{G}_{300} \mathrm{Ag}$. Every SEM image was averagely divided to 250 cells by the $50 \times 50$ grid. Inter-particle distance is defined as the smallest distance between the two particles in a cell. It increases with the flow rate of hydrogen ranging from 10 to $100 \mathrm{sccm}$ and decreases with further increase in the flow rate of hydrogen. The percentage of $\mathrm{G}_{100}-\mathrm{Ag}$ reaches a maximum value because it contains much more electronegative $\mathrm{C}-\mathrm{O}$ bonds to absorb $\mathrm{Ag}$ atoms or clusters to generate higher growth core density. These growth cores grow up and coalesce with each other to form irregular island shapes, which would contribute to narrowing the distances between $\mathrm{Ag}$ NPs to form hot spots, pointed out by the arrows in Fig. 3c. The percentage of $\mathrm{C}-\mathrm{O}$ bonds sharply decreases when the flow rate of hydrogen exceeds $100 \mathrm{sccm}$. Most $\mathrm{Ag}$ atoms or clusters independently grow into big NPs because of the less active sites density, which is affected by the $\mathrm{C}-\mathrm{O}$ bonds. It was considered that the maximum amount of hot spots would be generated on the sample $\mathrm{G}_{100}-\mathrm{Ag}$, which would give the strongest SERS single.

\subsection{Raman spectra for $G_{100}$ and $G_{100}-\mathbf{A g}$}

Fig. 4 shows the Raman spectra of $\mathrm{G}_{100}$ and $\mathrm{G}_{100} \mathrm{Ag}$ (formed by the deposition of $\mathrm{Ag}$ on $\mathrm{G}_{100}$ for $90 \mathrm{~s}$ ). It is shown that the sample $\mathrm{G}_{100}$ owns three graphene characteristic Raman bands: $\mathrm{D}, \mathrm{G}$, and $2 \mathrm{D}$ bands. By contrast, the sample $\mathrm{G}_{100}-\mathrm{Ag}$ owns not only the enhanced three Raman bands, but also the $\mathrm{D}^{\prime}$ band and another $\mathrm{D}+\mathrm{D}^{\prime}$ band. According to the research reported by Kalbac et al., ${ }^{41}$ the Raman signal of graphene is enhanced by energy transfer from surface plasmon resonance excited between Ag NPs. In addition, the doping of Ag NPs would induce the artificial defects in graphene, which result in the emergence of the $\mathrm{D}^{\prime}$ band and the enhancement of the $\mathrm{D}$ band. ${ }^{42}$ The $\mathrm{D}+\mathrm{D}^{\prime}$ band is due to two-phonon defect-assisted process. ${ }^{43}$ 

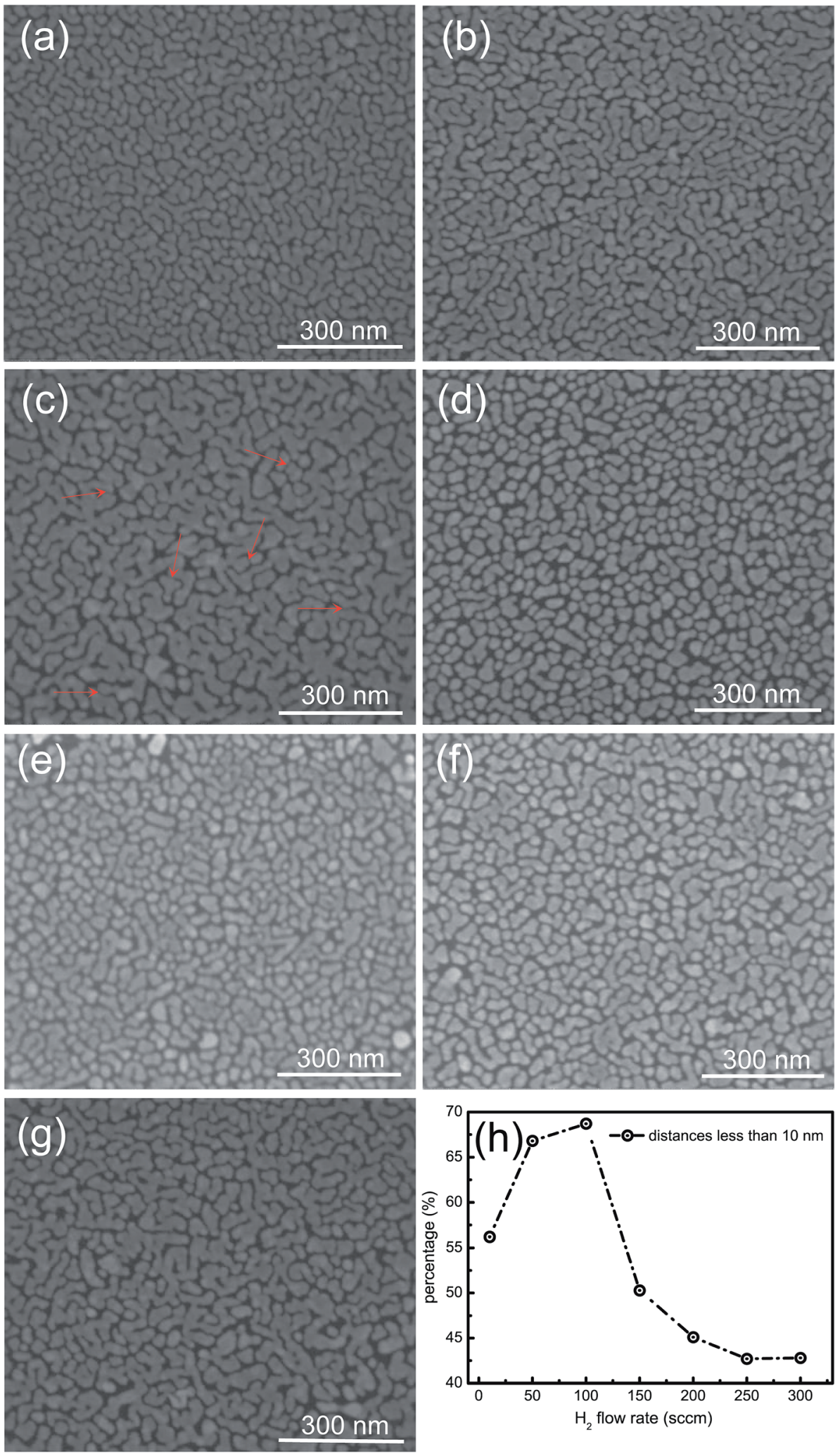

Fig. 3 (a-g) SEM images for Ag NPs on the samples of $G_{10}, G_{50}, G_{100}, G_{150}, G_{200}, G_{250}$ and $G_{300}$. (h) The percentage of distances between adjacent NPs less than $10 \mathrm{~nm}$ calculated from the corresponding SEM images. 


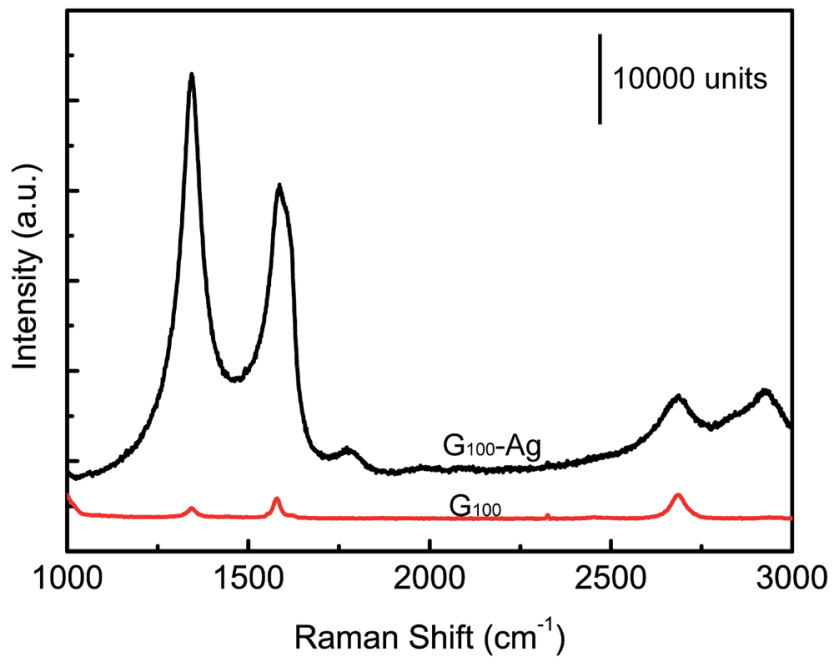

Fig. 4 Raman spectra for $\mathrm{G}_{100}$ and $\mathrm{G}_{100}-\mathrm{Ag}$.

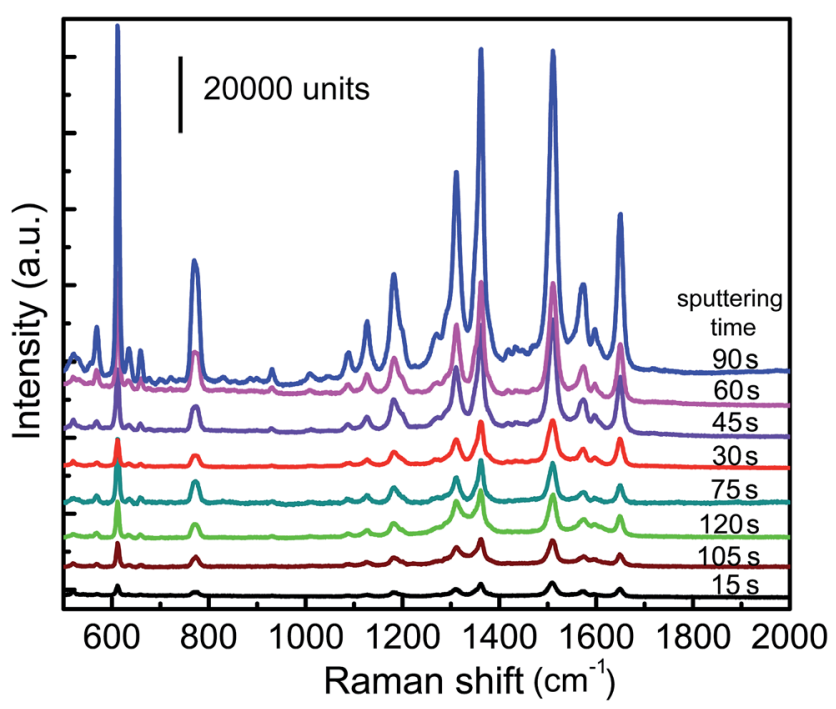

Fig. 5 Raman spectra of R6G molecules $\left(10^{-5} \mathrm{M}\right)$ on graphene $\left(\mathrm{G}_{10}\right)$ with Ag NPs prepared at different sputtering time.

\subsection{SERS spectra of R6G on Ag/graphene composites}

Fig. 5 shows that Raman spectra of R6G molecules $\left(10^{-5} \mathrm{M}\right)$ on the graphene substrate of the sample $\mathrm{G}_{10}$ with different characteristic Ag NPs, prepared by sputtering at different sputtering times ranging from 15 to $120 \mathrm{~s}$. It can be observed that Raman intensity shows an increase when the sputtering time is increased from 15 to $90 \mathrm{~s}$. However, the Raman intensity sharply decreases on the prolonging sputtering time. It is anticipated that the sizes of Ag NPs are small and the distances of Ag NPs are too large at shorter sputtering time, whereas the Ag NPs would be converted to continuous films when the sputtering time is very long. It is obvious that the Raman intensity shows a decrease when the sputtering time is either too short or too long. The Ag NPs prepared by sputtering for $90 \mathrm{~s}$ on uniform graphene show the strongest SERS signal.

$\mathrm{Ag}$ NPs were homogeneously sputtered on graphene sheets for an optimal depositing time of $90 \mathrm{~s}$. Fig. 6a shows the SERS spectra of R6G for different $\mathrm{Ag} / \mathrm{graphene}$ substrates including $\mathrm{G}_{10}-\mathrm{Ag}, \mathrm{G}_{50}-\mathrm{Ag}, \mathrm{G}_{100}-\mathrm{Ag}, \mathrm{G}_{150}-\mathrm{Ag}, \mathrm{G}_{250}-\mathrm{Ag}$, and $\mathrm{G}_{300}-\mathrm{Ag}$. The positions of the R6G characteristic peaks $1130 \mathrm{~cm}^{-1}, 1190$ $\mathrm{cm}^{-1}, 1360 \mathrm{~cm}^{-1}, 1509 \mathrm{~cm}^{-1}, 1575 \mathrm{~cm}^{-1}$, and $1650 \mathrm{~cm}^{-1}$ are generally in agreement with the previously reported measurement results. ${ }^{24,44}$ Amongst these samples, the sample $\mathrm{G}_{100} \mathrm{Ag}$ shows the strongest Raman intensity and the Raman intensity of sample $\mathrm{G}_{250} \mathrm{Ag}$ is the weakest compared with that of the other samples. The Raman intensity of $\mathrm{G}_{100} \mathrm{-Ag}$ is almost $0.5 \times$ $10^{3}$ times stronger than that of the sample $\mathrm{G}_{250} \mathrm{Ag}$. In general, SERS results from a combination of the EM and the CM. The EM plays a main role for SERS and it is originated from the morphology of the Ag NPs. The sample $\mathrm{G}_{100}$ contains much more $\mathrm{C}-\mathrm{O}$ bonds, which contribute to controlling the distribution of the Ag NPs covered on it. The percentage for distances less than $10 \mathrm{~nm}$ for the sample $\mathrm{G}_{100}-\mathrm{Ag}$ reaches a maximum value, and it would develop a maximum number of hot spots for SERS. The SERS EF change with the flow rate of hydrogen is shown in Fig. 6b. SERS EF is used to estimate the Raman enhancement effect amongst each sample. The SERS EF is calculated by the following formula: ${ }^{45}$
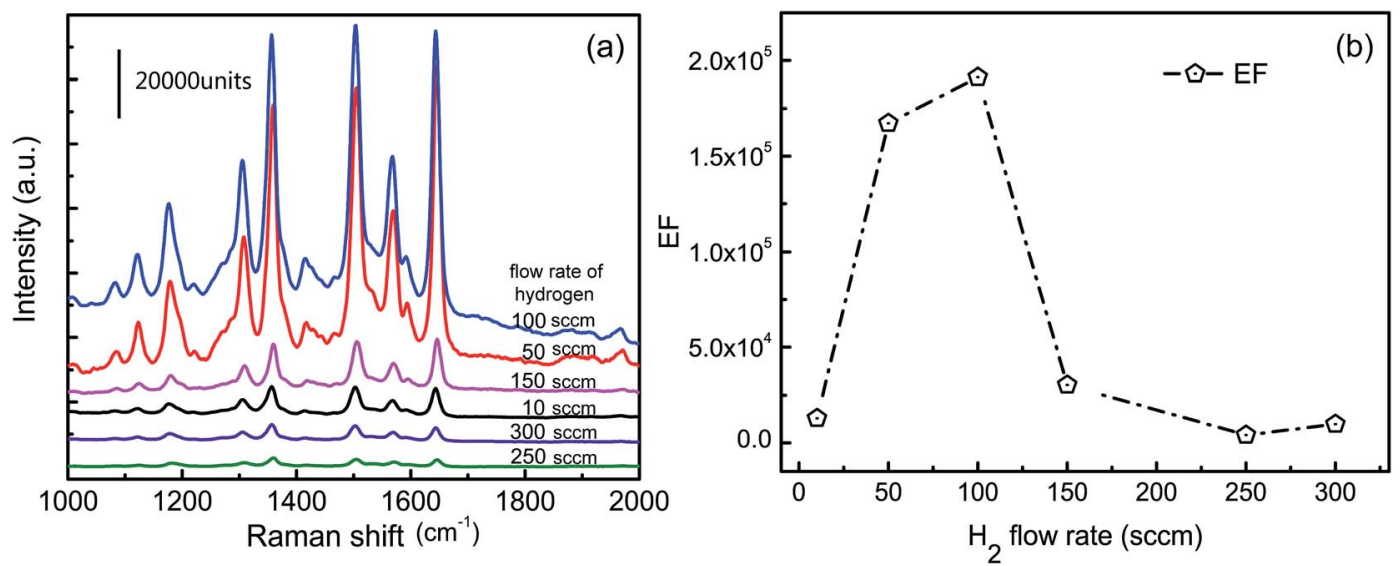

Fig. 6 (a) SERS spectra of $10^{-7}$ M R6G for various samples: $G_{10}-A g(90 \mathrm{~s}), G_{50}-A g(90 \mathrm{~s}), G_{100}-A g(90 \mathrm{~s}), G_{150}-A g(90 \mathrm{~s}), G_{250}-A g(90 \mathrm{~s})$, and $G_{300^{-}}$ $\mathrm{Ag}(90 \mathrm{~s})$. (b) The SERS enhancement factor (EF). 


$$
\mathrm{EF}=\frac{I_{\text {SERS }}}{I_{\text {bulk }}} \times \frac{N_{\text {bulk }}}{N_{\text {SERS }}}
$$

where $I_{\text {SERS }}$ is the intensity of the probe molecules dripped to the SERS substrate and $I_{\text {bulk }}$ is the intensity of the probe molecules dripped to the standard substrate. $N_{\text {SERS }}$ and $N_{\text {bulk }}$ are the number of probe molecules on the SERS substrate and standard substrate, respectively. The substrate with transferred graphene $\left(\mathrm{G}_{100}\right)$ but without $\mathrm{Ag}$ NPs was used as a standard substrate. It was observed that the EF was largest for the sample $\mathrm{G}_{100}-\mathrm{Ag}$ and smallest for the sample $\mathrm{G}_{250}-\mathrm{Ag}$. The variation tendency of the percentage for $\mathrm{C}-\mathrm{O}$ and the percentage for distances less than $10 \mathrm{~nm}$ between adjacent Ag NPs is similar to the SERS EF. This reveals that the percentage of $\mathrm{C}-\mathrm{O}$ in graphene would directly affect the distribution and morphology of the deposited Ag NPS on graphene and consequently the SERS intensity.

\section{Conclusion}

Graphene with different amounts of defects and layers was obtained by controlling the flow rate of hydrogen during the reacting process. The Ag NPs covered on the prepared graphene formed irregular island shapes, affected by the defects, which mainly consisted of $\mathrm{C}-\mathrm{O}$ bonds in graphene. It was considered that the $\mathrm{C}-\mathrm{O}$ bonds in graphene have the ability to provide active sites to absorb $\mathrm{Ag}$ atoms or clusters to create growth cores for Ag NPS, which would be developed to form hot spots for SERS. The SERS EF, the percentage of $\mathrm{C}-\mathrm{O}$ bonds, and the percentages of distances of less than $10 \mathrm{~nm}$ between adjacent $\mathrm{Ag}$ NPs have the similar variation trend. The $\mathrm{EF}$ of $\mathrm{G}_{100}-\mathrm{Ag}$ shows the maximum reaching $10^{5}$, which is almost $0.5 \times 10^{3}$ times stronger than that of the sample $\mathrm{G}_{250} \mathrm{Ag}$ having the weakest effect of SERS.

\section{Acknowledgements}

This work was supported by the National Natural Science Foundation of China (No. 51661145025, 11274055).

\section{References}

1 M. G. Albrecht and J. A. Creighton, J. Am. Chem. Soc., 1977, 99, 5215-5217.

2 D. L. Jeanmaire and R. P. Van Duyne, J. Electroanal. Chem. Interfacial Electrochem., 1977, 84, 1-20.

3 G. L. Liu, Y. Lu, J. Kim, J. C. Doll and L. P. Lee, Adv. Mater., 2005, 17, 2683-2688.

4 J. J. Baumberg, T. A. Kelf, Y. Sugawara, S. Cintra, M. E. Abdelsalam, P. N. Bartlett and A. E. Russell, Nano Lett., 2005, 5, 2262-2273.

5 J. F. Li, Y. F. Huang, Y. Ding, Z. L. Yang, S. B. Li, X. S. Zhou, F. R. Fan, W. Zhang, Z. Y. Zhou, D. Y. Wu, B. Ren, Z. L. Wang and Z. Q. Tian, Nature, 2010, 464, 392.

6 D.-K. Lim, K.-S. Jeon, H. M. Kim, J.-M. Nam and Y. D. Suh, Nat. Mater., 2010, 9, 60.
7 M. Fleischmann, P. J. Hendra and A. J. McQuillan, Chem. Phys. Lett., 1974, 26, 163-166.

8 A. Otto, I. Mrozek, H. Grabhorn and W. Akemann, J. Phys.: Condens. Matter, 1992, 4, 1143.

9 G. C. Schatz, M. A. Young and R. P. Duyne, Top. Appl. Phys., 2006, 103, 19-45.

10 E. C. L. Ru and P. G. Etchegoin, Principles of SurfaceEnhanced Raman Spectroscopy and Related Plasmonic Effect, 2009, vol. 47, pp. 415-464.

11 M. Udagawa, C. C. Chou, J. C. Hemminger and S. Ushioda, Phys. Rev. B: Condens. Matter Mater. Phys., 1981, 23, 68436846.

12 X. D. Jiang and A. Campion, Chem. Phys. Lett., 1987, 140, 95100.

13 M. Fan, G. F. S. Andrade and A. G. Brolo, Anal. Chim. Acta, 2011, 693, 7-25.

14 Y. Han, R. Lupitskyy, T. M. Chou, C. M. Stafford, H. Du and S. Sukhishvili, Anal. Chem., 2011, 83, 5873-5880.

15 S. Malynych and G. Chumanov, J. Am. Chem. Soc., 2003, 125, 2896-2898.

16 N. M. Kha, C. H. Chen and W. N. Su, Phys. Chem. Chem. Phys., 2015, 17, 21226-21235.

17 W. Xu, X. Ling, J. Xiao, M. S. Dresselhaus, J. Kong, H. Xu, Z. Liu and J. Zhang, Proc. Natl. Acad. Sci. U. S. A., 2012, 109, 9281-9286.

18 F. Bonaccorso, Z. Sun, T. Hasan and A. C. Ferrari, Nat. Photonics, 2010, 4, 611-622.

19 A. H. C. Neto, F. Guinea, N. M. R. Peres, K. S. Novoselov and A. K. Geim, Rev. Mod. Phys., 2009, 81, 109-162.

20 C. G. Lee, X. D. Wei, J. W. Kysar and J. Hone, Science, 2008, 321, 385-388.

21 G. Lu, H. Li, C. Liusman, Z. Yin, S. Wu and H. Zhang, Chem. Sci., 2011, 2, 1817-1821.

22 Y. Wang, Z. Ni, H. Hu, Y. Hao, C. P. Wong, T. Yu, J. T. L. Thong and Z. X. Shen, Appl. Phys. Lett., 2010, 97, 163111.

23 M. Losurdo, I. Bergmair, B. Dastmalchi, T.-H. Kim, M. M. Giangregroio, W. Jiao, G. V. Bianco, A. S. Brown, K. Hingerl and G. Bruno, Adv. Funct. Mater., 2014, 24, 1864-1878.

24 Y. Liu, Y. Hu and J. Zhang, J. Phys. Chem. C, 2014, 118, 89938998.

25 X. Li, W. C. H. Choy and X. Ren, Adv. Funct. Mater., 2014, 24, 3114-3122.

26 J. Leem, M. C. Wang and P. Kang, Nano Lett., 2015, 15. 27 X. Fu, L. Pan and S. Li, Appl. Surf. Sci., 2015, 363, 412-420.

28 X. Wang, G. Meng and C. Zhu, Adv. Funct. Mater., 2013, 23, 5771-5777.

29 C. Mattevi, H. Kim and M. Chhowalla, J. Mater. Chem., 2011, 21, 3324-3334.

30 X. Li, Science, 2009, 324, 1312-1314.

31 L. Xuesong, C. Weiwei and A. Jinho, Science, 2009, 324, 13121314.

32 X. Li, Y. Zhu and W. Cai, Nano Lett., 2009, 9, 4359-4363.

33 A. Umair and H. Raza, Nanoscale Res. Lett., 2012, 7, 1-5.

34 A. Das, B. Chakraborty and A. K. Sood, Bull. Mater. Sci., 2007, 31, 579-584. 
35 S. Hussain, M. W. Iqbal, J. Park, et al., Physical and electrical properties of graphene grown under different hydrogen flow in low pressure chemical vapor deposition, Nanoscale Res. Lett., 2014, 9(1), 546.

36 M. Kalbac, O. Frank and L. Kavan, The control of graphene double-layer formation in copper-catalyzed chemical vapor deposition, Carbon, 2012, 50(10), 3682-3687.

37 I. Vlassiouk, M. Regmi, P. Fulvio, et al., Role of hydrogen in chemical vapor deposition growth of large single-crystal graphene, ACS Nano, 2011, 5(7), 6069.

38 J. An, E. Voelkl, W. S. Ji, et al., Domain (Grain) Boundaries and Evidence of "Twinlike" Structures in Chemically Vapor Deposited Grown Graphene, ACS Nano, 2011, 5(4), 24332439.
39 J. H. Jang, J. C. Yoon and P. Thiyagarajan, $R S C A d v ., 2015,5$, 62772-62777.

40 Y. K. Yang, C. E. He and W. J. He, J. Nanopart. Res., 2011, 13, 1-11.

41 M. Kalbac, V. Vales and J. Vejpravova, The effect of a thin gold layer on graphene: A Raman spectroscopy study, $R S C$ Adv. , 2014, 4(105), 60929-60935.

42 H. Liu, S. Ryu, Z. Chen, et al., Photochemical Reactivity of Graphene, J. Am. Chem. Soc., 2009, 131(47), 17099-17101.

43 A. C. Ferrari and D. M. Basko, Raman spectroscopy as a versatile tool for studying the properties of graphene, Nat. Nanotechnol., 2013, 8(4), 235.

44 E. S. Thrall, A. C. Crowther and Y. Zhonghua, Nano Lett., 2012, 12, 1571-1577.

45 W. B. Cai, B. Ren and X. Q. Li, Surf. Sci., 1998, 406, 9-22. 